\title{
Erratum to: Ultra-High Field Proton MR Spectroscopy in Early- Stage Amyotrophic Lateral Sclerosis
}

\author{
Ian Cheong ${ }^{1} \cdot$ Małgorzata Marjańska $^{1} \cdot$ Dinesh K. Deelchand $^{1} \cdot$ Lynn E. Eberly $^{2}$ • \\ David Walk ${ }^{3}$ Gülin Ö $\mathbf{z}^{1}$
}

Published online: 18 May 2017

(C) Springer Science+Business Media New York 2017

\section{Erratum to: Neurochem Res \\ DOI 10.1007/s11064-017-2248-2}

The original version of this article unfortunately contained a mistake. The presentation of Table 1 was incorrect. The corrected Table 1 is given below. The original article was corrected.

The online version of the original article can be found under doi:10.1007/s11064-017-2248-2.

Ian Cheong

cheo0043@umn.edu

1 Department of Radiology, Center for Magnetic Resonance Research, University of Minnesota, 2021 6th St. S.E., Minneapolis, MN 55455, USA

2 Division of Biostatistics, School of Public Health, University of Minnesota, Minneapolis, USA

3 Department of Neurology, University of Minnesota, Minneapolis, USA 
Table 1 Clinical and ${ }^{1} \mathrm{H}-\mathrm{MRS}$ (7 T) data characteristics of scanned subjects by group and brain region examined

\begin{tabular}{|c|c|c|c|c|c|c|}
\hline & \multicolumn{3}{|l|}{ Motor cortex } & \multicolumn{3}{|l|}{ Pons } \\
\hline & $\operatorname{ALS}(\mathrm{N}=19)$ & Controls $(\mathrm{N}=17)$ & $P$-values & $\operatorname{ALS}(\mathrm{N}=15)$ & Controls $(\mathrm{N}=15)$ & $P$-values \\
\hline \multicolumn{7}{|l|}{ Clinical parameters } \\
\hline Sex ratio, male:female & $10: 9$ & $10: 7$ & $0.75^{*}$ & $8: 7$ & $8: 7$ & $1^{*}$ \\
\hline Age [range], years & $57 \pm 9[31-74]$ & $57 \pm 9[30-69]$ & 1 & $56 \pm 10[31-70]$ & $56 \pm 9[30-69]$ & 0.92 \\
\hline $\begin{array}{l}\text { Brain hemisphere scanned, } \\
\text { left:right }\end{array}$ & $8: 11$ & $8: 9$ & $1^{*}$ & N/A & N/A & \\
\hline Disease duration [range], months & $40.2 \pm 43.0[3.5-147.8]$ & N/A & & $37.0 \pm 40.2[3.5-147.8]$ & N/A & \\
\hline Site of onset, limb:bulbar & $15: 4$ & N/A & & $12: 3$ & N/A & \\
\hline $\begin{array}{l}\text { El escorial diagnosis (no. of } \\
\text { patients) }\end{array}$ & $\begin{array}{l}\text { Possible (7) } \\
\text { Probable (8) } \\
\text { Definite (4) }\end{array}$ & N/A & & $\begin{array}{l}\text { Possible (6) } \\
\text { Probable (7) } \\
\text { Definite (2) }\end{array}$ & N/A & \\
\hline Current riluzole use, yes:no & $8: 11$ & N/A & & $6: 9$ & N/A & \\
\hline ALSFRS-R score [range] & $39.8 \pm 5.6[26-45]$ & $48 \pm 0$ & $<0.001$ & $40.6 \pm 5.2[26-45]$ & $48 \pm 0$ & $<0.001$ \\
\hline UMN score [range] & $2.5 \pm 1.3[1-6]$ & N/A & & $2.3 \pm 1.1[1-5]$ & N/A & \\
\hline \multicolumn{7}{|l|}{7 T MRS quality parameters } \\
\hline Signal-to-noise ratio & $205 \pm 37$ & $201 \pm 35$ & 0.72 & $45 \pm 9$ & $53 \pm 17$ & 0.11 \\
\hline Water linewidth, $\mathrm{Hz}$ & $10 \pm 2$ & $11 \pm 2$ & 0.53 & $16 \pm 2$ & $15 \pm 2$ & 0.15 \\
\hline$\% \mathrm{CSF}$ & $11.3 \pm 5.3$ & $10.2 \pm 3.9$ & 0.55 & $0.9 \pm 0.3$ & $1.1 \pm 0.3$ & 0.26 \\
\hline
\end{tabular}

Data are given as mean \pm SD or counts

*Denotes $P$-values that are from Fisher's exact tests. Other $P$-values are from unpaired, two-tailed Student's t-tests 